\title{
White Matter Integrity, Duration of Untreated Psychosis, and Antipsychotic Treatment Response in Medication-Naïve First Episode Psychosis Patients
}

\author{
Nina Vanessa Kraguljac, MD², Thomas Anthony, MSEE², Charity Johanna Morgan, $\mathrm{PhD}^{3}$, \\ Ripu Daman Jindal, MD ${ }^{1,4}$, Mark Steven Burger, DO ${ }^{1}$, Adrienne Carol Lahti, MD1 \\ ${ }^{1}$ Department of Psychiatry and Behavioral Neurobiology, University of Alabama at Birmingham \\ 2Department of Electrical and Computer Engineering/ IT Research Computing, University of \\ Alabama at Birmingham \\ ${ }^{3}$ Department of Biostatistics, University of Alabama at Birmingham \\ ${ }^{4}$ Department of Neurology, Birmingham VA Medical Center
}

\begin{abstract}
It is becoming increasingly clear that longer duration of untreated psychosis (DUP) is associated with adverse clinical outcomes in patients with psychosis spectrum disorders. Because this association is often cited when justifying early intervention efforts, it is imperative to better understand underlying biological mechanisms. We enrolled 66 antipsychotic-naïve first episode psychosis (FEP) patients and 45 matched healthy controls in this trial. At baseline, we used a human connectome style diffusion weighted imaging (DWI) sequence to quantify white matter integrity in both groups. Patients then received 16 weeks of treatment with risperidone, 51 FEP completed the trial. We compared whole brain fractional anisotropy (FA), mean diffusivity, axial diffusivity (AD) and radial diffusivity between groups. To test if structural white matter integrity mediates the relationship between longer DUP and poorer treatment response, we fit a mediator model and estimated indirect effects. We found decreased whole brain FA and AD in medication-naive FEP compared to controls. In patients, lower FA was correlated with longer DUP $(r=-0.32 ; p=0.03)$ and poorer subsequent response to antipsychotic treatment $(r=0.40$; $\mathrm{p}=0.01$ ). Importantly, we found a significant mediation effect for FA (indirect effect: -2.70 ; $p=0.03$ ), indicating that DUP exerts its effects on treatment response through affecting white matter integrity. Our data provide empirical support to the idea the DUP may have fundamental pathogenic effects on the natural history of psychosis, suggest a biological mechanism underlying this phenomenon, and underscore the importance of early intervention efforts in this disabling neuropsychiatric syndrome.
\end{abstract}

Trial registration: ClinicalTrials.gov: NCT02034253

Users may view, print, copy, and download text and data-mine the content in such documents, for the purposes of academic research, subject always to the full Conditions of use:http://www.nature.com/authors/editorial_policies/license.html\#terms

Corresponding Author: Nina Vanessa Kraguljac, MD, Department of Psychiatry and Behavioral Neurobiology, The University of Alabama at Birmingham, SC 501, 1720 7th Ave S, Birmingham, AL 35294-0017, 205-996-7171, nkraguljac@uabmc.edu. 


\section{INTRODUCTION}

In patients with a psychosis spectrum disorder, the median duration of untreated psychosis (DUP), defined as the time between first occurrence of positive symptoms and initial antipsychotic treatment, is approximately 74 weeks in the US ${ }^{1}$. It is becoming clear that longer DUP is associated with adverse clinical outcomes ${ }^{2,3}$, and may even be a determinant of overall outcomes. It has been highlighted that the long DUP may be one of the least understood but most important public mental health problems in our society today ${ }^{4}$, this is true particularly when it comes to the neurobiological underpinnings of this phenomenon. Especially because the relationship between longer DUP and poorer overall outcomes is often cited when justifying early intervention efforts, and because an unequivocal proof of a mechanistic link would have important implications for the use of treatment in case of the patient's refusal ${ }^{5}$, it is imperative to better understand putative biological mechanisms underlying this association.

However, the idea that untreated psychosis may adversely impact brain function and structure remains debated ${ }^{6,7}$ and only limited empirical evidence is available. To our knowledge, two studies thus far have demonstrated a link between functional brain connectivity, DUP, and antipsychotic treatment response ${ }^{8,9}$. Importantly, both found that connectivity mediated the relationship between longer DUP and poorer subsequent treatment response, supporting the idea that untreated psychosis adversely affects the brain in a clinically relevant manner. Because functional connectivity can be perturbed by a number of pathological states such as disruptions in various neurotransmitter systems ${ }^{10-12}$ or deficits in the white matter tracts ${ }^{13}, 14$ that provide the structural framework of functional brain networks ${ }^{15,16}$, a logical expansion of this line of research is to investigate a presumed role of structural connectivity in the relationship between DUP and treatment response.

Here, we enrolled a group of antipsychotic-naïve first episode psychosis patients (FEP) in a longitudinal diffusion weighted imaging (DWI) study to test the hypothesis that lower white matter integrity at baseline is associated with longer DUP and poorer clinical response after sixteen weeks of antipsychotic treatment. We chose this timeframe because longer treatment trials are warranted in $\mathrm{FEP}^{17}$ and early symptom reduction does not appear to be a clinically useful predictor of antipsychotic treatment response in this population ${ }^{18}$. Our primary variable of interest was whole brain white matter fractional anisotropy (FA), a dimensionless summary measure of microstructural white matter integrity. We chose to focus on whole brain white matter integrity, as we and others have found that whole brain white matter integrity was predictive of subsequent response to antipsychotic treatment in schizophrenia spectrum patients ${ }^{19,20}$. As secondary analyses, we also quantified mean diffusivity (MD), radial diffusivity (RD), and axial diffusivity (AD), three related measures that capture various aspects of diffusivity. We then conducted mediation analyses to test if white matter integrity is a mediator in the relationship between longer DUP and poorer response to antipsychotic treatment. 


\section{MATERIALS AND METHODS}

FEP were recruited from outpatient, inpatient and emergency room settings at the University of Alabama at Birmingham (UAB). Healthy controls (HC) matched on age, sex and parental occupation were recruited by advertisements. Written informed consent was obtained (once FEP were deemed to have capacity ${ }^{21}$ ) prior to enrollment in this UAB Institutional Review Board approved study.

Participants were excluded if they had major neurological or medical conditions, history of head trauma with loss of consciousness, substance use disorders (excluding nicotine and cannabis) within one month of imaging, were pregnant or breastfeeding, or had MRI contraindications. Patients were either antipsychotic-naïve or had no more than five days of lifetime antipsychotic exposure prior to study entry ( $83 \%$ of patients did not receive any antipsychotic prior to the initial scan). Controls with a personal history of a mental illness or family history in a first-degree relative of a psychotic disorder were excluded.

Diagnoses were established by consensus of two board certified psychiatrists (ACL and NVK) taking into consideration information from the Diagnostic Interview for Genetic Studies (DIGS) or Mini-International Neuropsychiatric Interview (MINI) and medical records as available. The Brief Psychiatric Rating Scale (BPRS) and Repeatable Battery for the Assessment of Neuropsychological Status (RBANS) were used to assess symptom severity and cognition, respectively. We operationally defined DUP as the time between first onset of positive symptoms and the start of treatment (the time at which the first antipsychotic prescription was written, which also coincided with enrollment in the study). The time of symptom onset was determined in consensus by two psychiatrists (ACL and NVK) via clinical interviews with the patient and family members as well as medical records (psychiatric assessment, review of systems, family history, laboratory workup) and clinical observations and assessments over several months of follow up.

FEP enrolled in this longitudinal study entered into a sixteen week trial of oral risperidone using a flexible dosing regimen (Figure 1). Risperidone was started at $0.5-1 \mathrm{mg}$ and titrated in 1-2mg increments; dosing was based on therapeutic and side effects. In case of excessive side effect burden, as determined by a study physician, patients were switched to aripiprazole started at $2-5 \mathrm{mg}$ and titrated in $2.5-10 \mathrm{mg}$ increments (two patients were switched to aripiprazole; one developed hyperprolactinemia within the first week of using risperidone, the second was switched after seven weeks of treatment due to irritability). Use of concomitant medication was permitted as clinically indicated (20 patients were prescribed benztropine, 14 an SSRI, 5 trazodone, 1 buproprione, 1 lithium, 1 valproic acid, 1 prazosin, 1 stimulant). Treatment response (i.e. percent change in symptom severity) was calculated as follows: [(BPRS positive score at baseline - BPRS positive score at endpoint)/ BPRS positive score at baseline] $* 100$.

\section{Data acquisition}

Imaging was performed on a 3T Siemens Magnetom Prisma scanner equipped with a 20-channel head coil. A T2 weighted scan was acquired for anatomical reference (TR/TE: 3200/ 563ms; GRAPPA factor 2; slice thickness $0.8 \mathrm{~mm}$; 208 slices, voxel size $0.8 \mathrm{~mm}^{3}$ ). 
DWI data was acquired with opposing phase encoding directions (anterior $>$ posterior, and posterior > anterior; [TR/TE: $3230 \mathrm{~ms} / 89.20 \mathrm{~ms}$; multiband acceleration factor 4, Flip angle: $84^{\circ}$; slice thickness $1.5 \mathrm{~mm}, 92$ slices, voxel size $1.5 \mathrm{~mm}^{3}, 92$ diffusion weighted images distributed equally over 2 shells with b-values of $\sim 1500 \mathrm{~s} / \mathrm{mm}^{2}$ and $\sim 3000 \mathrm{~s} / \mathrm{mm}^{2}$, as well as 7 interspersed $\mathrm{b}=\sim 0 \mathrm{~s} / \mathrm{mm}^{2}$ images]).

\section{Data preprocessing}

Preprocessing of DWI images was performed in TORTOISE (version 3.1.2) 22,23 . This included correction for thermal noise ${ }^{24}$, Gibbs-ringing ${ }^{25}$, high b-value based bulk motion and eddy-current distortions using a MAP-MRI model ${ }^{26,27}$, resampling of images to $1 \mathrm{~mm}^{3}$, and rotation of gradient tables independently for each DWI phase encoding direction with DIFF-PREP. Then, DR-BUDDI was used to correct EPI distortions with input from the anatomical image and to combine the two datasets using geometric averaging to generate the corrected dataset ${ }^{28}$. Tensors were computed with DIFF_CALC using a linear fitting algorithm. To spatially normalize images to the Illinois Institute of Technology atlas (IIT4) space, we implemented an optimized non-linear image registration procedure using a modified version of $3 \mathrm{dQwarp}$ in $\mathrm{AFNI}^{29}$ (Figure 2). As our primary variable of interest, we extracted whole brain white matter FA. To do this, we created a whole brain white matter mask based on the atlas image and extracted an averaged FA value for each participant, this was done on in standard space. We also calculated whole brain MD, AD, and RD.

\section{Data quality control}

We verified protocol adherence and visually inspected raw images for signal loss and artifacts. We then assessed bulk motion and excluded datasets with an average root mean square of absolute displacement $\left(\mathrm{RMS}_{\mathrm{abs}}\right)$ of greater than the voxel edge length $(1.5 \mathrm{~mm})$ or root mean square of relative displacement $\left(\mathrm{RMS}_{\text {rel }}\right)$ of $>0.05 \mathrm{~mm}$ averaged across phase encoding directions from further analyses. DR_BUDDI outputs were assessed for quality of motion, eddy current and EPI distortion correction, and scalar diffusion maps were inspected for anomalies in parameters.

\section{Statistical analyses}

We compared baseline differences in whole brain white matter integrity between FEP and $\mathrm{HC}$ using univariate analysis of variance with age, sex, and $\mathrm{RMS}_{\text {rel }}$ as covariates. We also examined the relationship between whole brain white matter FA and log transformed DUP as well as treatment response after 16 weeks in FEP using partial correlations with age, gender and $\mathrm{RMS}_{\text {rel }}$ as covariates. We then repeated analyses with whole brain MD, AD, and $\mathrm{RD}$.

To test if structural white matter integrity mediates the relationship between longer DUP and poorer treatment response, we used the method described by Preacher and Hayes ${ }^{30}$ to fit a mediator model and estimate indirect effects. The model controlled for age, sex and $\mathrm{RMS}_{\text {rel }}$. Accelerated bias-corrected bootstrap confidence intervals were used to test for statistical significance and to form $95 \%$ confidence intervals (based on 1000 replications) for the indirect effects ${ }^{31}$. 
As a post hoc analyses, we examined relationships between whole brain voxel-wise FA, $\mathrm{MD}, \mathrm{RD}$, and AD and DUP as well as treatment response using AFNI's 3dttest++; covariates included age, gender, $\mathrm{RMS}_{\text {rel }}$. Clustsim, a randomization/ permutation simulation to produce 10,000 iterations of noise only generated t-tests and determine global cluster-level threshold values, was implemented to control for the false positive rate (voxelwise threshold Bonferroni corrected $\mathrm{p}=.00625$ [accounting for 8 comparisons at $\mathrm{p}_{\text {uncorrected }}=.05$ ]; cluster threshold $a=0.05)^{32}$.

The sample here has no overlap with our previous studies on white matter integrity in psychosis spectrum patients ${ }^{19,33,34}$.

\section{RESULTS}

\section{Participant characteristics}

Of the 183 patients assessed for eligibility between June 2016 and April 2019, 74 FEP were consented and 66 were entered into the sixteen week trial of risperidone (Figure 2), a total of 51 FEP completed the study. We also enrolled $45 \mathrm{HC}$.

Groups did not differ in gender, age or parental socioeconomic status (Table). In patients, BPRS scores significantly decreased after 16 weeks of treatment, the average daily dose of risperidone at that time was $4.11+/-2.66 \mathrm{mg}$. Importantly, longer DUP predicted worse subsequent response to antipsychotic treatment $(r=-0.37 ; \mathrm{p}<0.01)$, but DUP was not associated with RBANS scores.

\section{Whole brain diffusion, DUP and treatment response}

Imaging data from $3 \mathrm{HC}$ and $10 \mathrm{FEP}$ did not pass quality control. Additionally, 4 FEP terminated the scan before DWI images were acquired. In summary, diffusion datasets from $52 \mathrm{FEP}$ and $42 \mathrm{HC}$ were included in analyses for group comparisons. Whole brain FA and AD were significantly lower in FEP compared to controls (FA: $F=8.58$; $<<0.01$; AD: $F=$ 23.98; $\mathrm{p}<0.01$; Figure $3 \mathrm{~A}$ ), but MD and RD did not differ between groups. There was no difference in whole brain FA, MD, RD or AD between patients who tested positive and negative for cannabis (all $\mathrm{p}>0.70$ ).

In FEP, lower FA was correlated with longer DUP $(r=-0.32 ; \mathrm{p}=0.03)$ and poorer antipsychotic treatment response $(r=0.40 ; p=0.01$; Figure $3 \mathrm{~B})$. We did not observe associations between $\mathrm{AD}, \mathrm{MD}$ or RD and DUP, treatment response, or RBANS scores.

As a post-hoc analysis, we also examined regional patterns of the relationships between DUP/ treatment response and white matter integrity in FEP. Longer DUP was associated with lower FA in the anterior corona radiata, but no regional patterns were seen with AD, $\mathrm{MD}$, or RD. Better subsequent response to antipsychotic treatment was associated with lower MD and RD in the inferior longitudinal fasciculus/ inferior fronto-occipital fasciculus (Figure 4). 


\section{Mediation Analyses}

The independent variable DUP predicted the dependent variable treatment response, DUP was correlated with the putative mediator FA, and FA predicted treatment response, thus fulfilling the prerequisites for testing a mediation effect ${ }^{35}$ (Figure 3C illustrates standardized regression coefficients). We did find a significant mediation effect for FA [indirect effect: $-2.70 ; p=0.03 ; 95 \%$ CI: $(-\infty,-0.25)$ ], indicating that DUP exerts its effects on treatment response through affecting white matter integrity.

\section{DISCUSSION}

To our knowledge, this is the first study to examine a putative role of white matter integrity in the observed association between DUP and clinical outcomes in FEP. We report evidence of white matter alterations in medication-naive FEP compared to controls, and demonstrate empirical links between the extent of FA alterations, DUP, and clinical response after sixteen weeks of antipsychotic treatment. Mediation analyses indicate that longer DUP impacts white matter integrity, which in turn influences clinical outcomes. Taken together, our data are consistent with the idea that the DUP may have fundamental pathogenic effects on the natural history of psychosis, suggest a biological mechanism underlying this phenomenon, and underscore the importance of early intervention efforts in this disabling neuropsychiatric syndrome.

Here, we found that whole brain FA and AD were decreased in FEP compared to controls, which is consistent with reports of widespread white matter integrity deficits in the schizophrenia literature ${ }^{36}$. However, we did not find alterations in MD or RD, which are often interpreted as evidence of neuroinflammation and myelin damage/ abnormal fiber crossing respectively.

Our findings replicate reports of an association between DUP and antipsychotic treatment response in psychosis spectrum patients ${ }^{37}$, and extend the literature by demonstrating that whole brain white matter integrity deficits in medication-naïve FEP appear to be more prominent as the DUP increases. While this contradicts several literature reviews ${ }^{6,}, 38$ failing to demonstrate unequivocal evidence of a relationship between DUP brain structure, it is important to note that these reviews solely focused on gray matter morphology. Unfortunately, data on white matter are scarce; we found only two other relevant publications ${ }^{39,}{ }^{40}$. In a letter to the editors, Lee and colleagues found an inverse relationship between DUP and FA in the left tapetum in a large group of medicated FEP with an average DUP of 14.6 weeks ${ }^{39}$, which is consistent with our findings. The other study was conducted in 43 medication-naïve FEP and 17 controls. Results showed a mix of areas with increased and decreased FA in patients compared to controls, and a correlation between DUP and $\mathrm{FA}^{40}$. It is notable that all three studies found an association between DUP and white matter integrity. The discrepancy between studies examining gray matter and those investigating white matter is perhaps not surprising, as trajectories of gray and white matter deterioration in schizophrenia have found to be dissimilar ${ }^{41}$. Post-hoc voxelwise analyses showed an association between DUP and FA in the anterior corona radiata, one of the brain areas with the largest effect sizes in terms of FA reductions in schizophrenia ${ }^{36}$. Consistent with our findings, Lee and colleagues reported a negative relationship between DUP and FA in the 
left anterior and right posterior corona radiata and the left internal capsule ${ }^{42}$. Similarly, in bipolar disorder, a negative relationship between FA in the anterior corona radiata and illness duration has been demonstrated, however, this was not found in schizophrenia patients ${ }^{43}$. The spatial extent of voxelwise associations with DUP was limited, which at first glance may be surprising given the finding of a significant negative correlation with whole brain FA. It is possible that existing subtle associations across white matter tracts did not survive the voxelwise threshold for multiple comparison corrections, suggesting that a whole brain approach may have greater power to detect relationships between imaging and clinical variables.

Our study also adds to a growing literature highlighting white matter integrity as a predictor of antipsychotic treatment response in patients with psychosis spectrum disorders. Similar to us, others have found more widespread FA decreases in FEP with a subsequent poor response compared to those with a good response ${ }^{44}$ and that baseline global white matter network organization showed greater alterations in FEP who subsequently showed poor treatment response compared to those with a more favorable response ${ }^{20}$. Our group previously found that whole brain orientation dispersion index (ODI), a measure of white matter fiber uniformity, was predictive subsequent treatment response in psychosis spectrum patients ${ }^{19}$.

Tying together our main findings, mediation analyses demonstrated an indirect effect of FA on the relationship between DUP and treatment response. In other words, our data suggests a process, i.e. white matter deterioration, through which DUP affects antipsychotic treatment response, which is consistent with the idea that DUP has fundamental pathogenic effects on the brain. Our findings are also in agreement with two studies examining a possible mediating role of functional brain connectivity in the association between these two variables. Sarpal and colleagues investigated functional connectivity of the striatum, a principal site of antipsychotic drug action ${ }^{9}$. They describe a significant mediation effect, indicating that DUP exerts it effects on treatment response trough corticostriatal connectivity. Our group recently reported that resting state connectivity of the default mode network in medication-naïve FEP mediates the relationship between DUP and subsequent response to antipsychotic medication ${ }^{8}$. Taken together, all three studies lend empirical support to the idea that untreated psychosis impacts brain connectivity in a clinically meaningful way and highlight neurobiological substrates underlying this phenomenon.

A number of mechanisms through with active psychosis may adversely affect the brain have been proposed. Several authors suggested that dopaminergic hyperactivity, whereby a prolonged elevation of dopamine during a psychotic episode, could lead to a progressive decline in brain volume in form of neuronal injury, which could potentially be reversed with antipsychotic medication treatment ${ }^{45}, 46$. McGlashan postulated that the DUP may be important in the sense that first episode patients with a long DUP may be well beyond the period of active deficit processes formation in their brain, while patients with short DUP may still have actively destructive neurobiological changes that can be arrested or reversed with antipsychotic treatment ${ }^{4}$. Interestingly, preclinical data shows that dopamine signaling via dopamine D2 receptors regulates myelination in response to chronic stress ${ }^{47}$ and that alterations in white matter are associated with defects in the dopaminergic system ${ }^{48}$. 
It is tempting to speculate a dopaminergic hyperactivity in FEP may result in reversible structural injury, but if it is prolonged, may permanently affect white matter integrity. In that case, those with a short DUP and little appreciable white matter deficits should respond well to antipsychotic medication, while those with a longer DUP should show greater white matter impairments and poorer response to treatment, which is consistent with our empirical findings. Another mechanism through which untreated psychosis could adversely affect the brain is altered glutamate metabolism, which has been reported in acutely ill early stage psychosis spectrum patients ${ }^{49}, 50$, and may be normalized after successful antipsychotic treatment ${ }^{50-53}$. Because glial cells and axons are vulnerable to glutamate related excitotoxicity 54,55 , it is conceivable that prolonged exposure to excess glutamate metabolism as the DUP increases, contributes to progressive white matter pathology and poorer overall outcomes in this patient population. It is intriguing that proposed mechanisms underlying the adverse impacts of DUP on the brain have in common an element where an initial structural injury is potentially reversible with antipsychotic treatment, but with prolonged exposure the pathological state becomes permanent and results in poor overall outcomes. Regardless of the underlying mechanisms, our data underscore the importance of early intervention efforts aimed at arresting DUP's detrimental effects on the brain.

One of the major strengths was that we enrolled FEP at first treatment contact mitigating confounds of antipsychotic medication exposure and illness chronicity. Even though this is a notoriously challenging patient population to recruit, our sample represents perhaps one of the largest antipsychotic-naïve first episode psychosis cohorts followed longitudinally in the US. We treated patients with risperidone, the chosen trial duration of 16 weeks allowed us to capture those who respond only after prolonged treatment reducing the possibility of late responders being incorrectly characterized as poor responders. Our retention rates were excellent for a clinical trial with antipsychotic medications, with only $\sim 23 \%$ attrition $^{56}$. We determined DUP using a clinical interview. A quantitative review comparing methods of gauging DUP concluded that clinical interviews are no less reliable than standardized assessment tools, but that the definition of treatment onset as first-ever antipsychotic medication prescription or first hospitalization may have greater validity compared to other methods such as first adequate response to treatment ${ }^{57}$. We enrolled subjects with a first psychotic episode, regardless of diagnosis. A subset of our patients were diagnosed with a mood disorder with psychotic features or with unspecified psychosis. It is possible that DUP has different effects depending on the primary diagnosis, but we were not adequately powered to test this. Future large-scale studies should investigate if DUP is factor affecting brain structure irrespective of the clinical diagnosis or if there are differential effects. We chose not to exclude patients who used cannabis. Though exposure to cannabis may affect brain structure and function, it is one of the major risk factors for developing a psychotic illness and therefore highly clinically relevant. Excluding patients with a history of cannabis use would have inadvertently biased our sample and limit the generalizability of our data. Finally, we obtained diffusion data with a human connectome style sequence and used state of the art data post-processing strategies including stringent quality control. This allowed us to acquire data with good spatial resolution, minimize distortions and other artifacts and accurately align corresponding brain areas across subjects. 
In conclusion, DUP has been identified as one most important public mental health problems today ${ }^{4}$. Our data are consistent with the idea the DUP may have fundamental pathogenic effects on the structural connectome which results in poorer clinical outcomes. Importantly, findings underscore the critical need of early intervention in an effort to alleviate disease burden and improve outcomes in this disabling neuropsychiatric syndrome.

\section{Acknowledgments}

\section{CONFLICT OF INTEREST}

This work was supported by the National Institute of Mental Health (R01MH112800 and R01MH102951, ACL; K23MH106683, NVK). We would like to thank UAB IT Research Computing for providing the HPC resources (compute, storage and networking) for this project. Cheaha is supported in part by the National Science Foundation under Grant No. OAC-1541310, the University of Alabama at Birmingham, and the Alabama Innovation Fund.

ACL has received an investigator initiated grant from Janssen Pharmaceuticals. All other authors declare no conflicts of interest, including relevant financial interests, activities, relationships, and affiliations.

\section{REFERENCES}

1. Kane JM, Robinson DG, Schooler NR, Mueser KT, Penn DL, Rosenheck RA et al. Comprehensive Versus Usual Community Care for First-Episode Psychosis: 2-Year Outcomes From the NIMH RAISE Early Treatment Program. Am J Psychiatry 2016; 173(4): 362-372. [PubMed: 26481174]

2. Primavera D, Bandecchi C, Lepori T, Sanna L, Nicotra E, Carpiniello B. Does duration of untreated psychosis predict very long term outcome of schizophrenic disorders? results of a retrospective study. Ann Gen Psychiatry 2012; 11(1): 21. [PubMed: 22856624]

3. Albert N, Melau M, Jensen H, Hastrup LH, Hjorthoj C, Nordentoft M. The effect of duration of untreated psychosis and treatment delay on the outcomes of prolonged early intervention in psychotic disorders. NPJ Schizophr 2017; 3(1): 34. [PubMed: 28951544]

4. McGlashan TH. Duration of untreated psychosis in first-episode schizophrenia: marker or determinant of course? Biol Psychiatry 1999; 46(7): 899-907. [PubMed: 10509173]

5. Anderson KK, Voineskos A, Mulsant BH, George TP, McKenzie KJ. The role of untreated psychosis in neurodegeneration: a review of hypothesized mechanisms of neurotoxicity in firstepisode psychosis. Can J Psychiatry 2014; 59(10): 513-517. [PubMed: 25565683]

6. Rund BR. Does active psychosis cause neurobiological pathology? A critical review of the neurotoxicity hypothesis. Psychol Med 2014; 44(8): 1577-1590. [PubMed: 24067164]

7. Wyatt RJ. Neuroleptics and the natural course of schizophrenia. Schizophr Bull 1991; 17(2): 325 351. [PubMed: 1679255]

8. Maximo JO, Nelson EA, Armstrong WP, Kraguljac NV, Lahti AC. Duration of untreated psychosis correlates with brain connectivity and morphology in medication-naive patients with first episode psychosis. Biological Psychiatry: CNNI in press.

9. Sarpal DK, Robinson DG, Fales C, Lencz T, Argyelan M, Karlsgodt KH et al. Relationship between Duration of Untreated Psychosis and Intrinsic Corticostriatal Connectivity in Patients with Early Phase Schizophrenia. Neuropsychopharmacology 2017; 42(11): 2214-2221. [PubMed: 28294137]

10. Kraguljac NV, Frolich MA, Tran S, White DM, Nichols N, Barton-McArdle A et al. Ketamine modulates hippocampal neurochemistry and functional connectivity: a combined magnetic resonance spectroscopy and resting-state fMRI study in healthy volunteers. Mol Psychiatry 2017; 22(4): 562-569. [PubMed: 27480494]

11. Ghisleni C, Bollmann S, Poil SS, Brandeis D, Martin E, Michels L et al. Subcortical glutamate mediates the reduction of short-range functional connectivity with age in a developmental cohort. $\mathrm{J}$ Neurosci 2015; 35(22): 8433-8441. [PubMed: 26041912]

12. Conio B, Martino M, Magioncalda P, Escelsior A, Inglese M, Amore M et al. Opposite effects of dopamine and serotonin on resting-state networks: review and implications for psychiatric disorders. Mol Psychiatry 2020; 25(1): 82-93. [PubMed: 30953003] 
13. Duerden EG, Halani S, Ng K, Guo T, Foong J, Glass TJA et al. White matter injury predicts disrupted functional connectivity and microstructure in very preterm born neonates. Neuroimage Clin 2019; 21: 101596. [PubMed: 30458986]

14. Au Duong MV, Audoin B, Boulanouar K, Ibarrola D, Malikova I, Confort-Gouny S et al. Altered functional connectivity related to white matter changes inside the working memory network at the very early stage of MS. J Cereb Blood Flow Metab 2005; 25(10): 1245-1253. [PubMed: 15843789]

15. Mollink J, Smith SM, Elliott LT, Kleinnijenhuis M, Hiemstra M, Alfaro-Almagro F et al. The spatial correspondence and genetic influence of interhemispheric connectivity with white matter microstructure. Nat Neurosci 2019; 22(5): 809-819. [PubMed: 30988526]

16. Skudlarski P, Jagannathan K, Calhoun VD, Hampson M, Skudlarska BA, Pearlson G. Measuring brain connectivity: diffusion tensor imaging validates resting state temporal correlations. Neuroimage 2008; 43(3): 554-561. [PubMed: 18771736]

17. Emsley R, Rabinowitz J, Medori R. Time course for antipsychotic treatment response in firstepisode schizophrenia. Am J Psychiatry 2006; 163(4): 743-745. [PubMed: 16585455]

18. Gallego JA, Robinson DG, Sevy SM, Napolitano B, McCormack J, Lesser ML et al. Time to treatment response in first-episode schizophrenia: should acute treatment trials last several months? J Clin Psychiatry 2011; 72(12): 1691-1696. [PubMed: 21939612]

19. Kraguljac NV, Anthony T, Monroe WS, Skidmore FM, Morgan CJ, White DM et al. A longitudinal neurite and free water imaging study in patients with a schizophrenia spectrum disorder. Neuropsychopharmacology 2019; 44(11): 1932-1939. [PubMed: 31153156]

20. Crossley NA, Marques TR, Taylor H, Chaddock C, Dell'Acqua F, Reinders AA et al. Connectomic correlates of response to treatment in first-episode psychosis. Brain 2017; 140(2): 487-496. [PubMed: 28007987]

21. Carpenter WT Jr., Gold JM, Lahti AC, Queern CA, Conley RR, Bartko JJ et al. Decisional capacity for informed consent in schizophrenia research. Arch Gen Psychiatry 2000; 57(6): 533-538. [PubMed: 10839330]

22. Pierpaoli C, Walker L, Irfanoglu MO, Barnett A, Basser P, Chang L-C et al. TORTOISE: an integrated software package for processing of diffusion MRI data. ISMRM 18th annual meeting: Stockholm, Sweden, 2010.

23. TORTOISEv3:Improvements and new features of the NIH diffusion MRI processing pipeline. Proceedings of the ISMRM 25th annual meeting 2017; Honolulu, HI.

24. Veraart J, Fieremans E, Novikov DS. Diffusion MRI noise mapping using random matrix theory. Magn Reson Med 2016; 76(5): 1582-1593. [PubMed: 26599599]

25. Kellner E, Dhital B, Kiselev VG, Reisert M. Gibbs-ringing artifact removal based on local subvoxel-shifts. Magn Reson Med 2016; 76(5): 1574-1581. [PubMed: 26745823]

26. Rohde GK, Barnett AS, Basser PJ, Marenco S, Pierpaoli C. Comprehensive approach for correction of motion and distortion in diffusion-weighted MRI. Magn Reson Med 2004; 51(1): 103-114. [PubMed: 14705050]

27. Ozarslan E, Koay CG, Shepherd TM, Komlosh ME, Irfanoglu MO, Pierpaoli C et al. Mean apparent propagator (MAP) MRI: a novel diffusion imaging method for mapping tissue microstructure. Neuroimage 2013; 78: 16-32. [PubMed: 23587694]

28. Irfanoglu MO, Modi P, Nayak A, Hutchinson EB, Sarlls J, Pierpaoli C. DR-BUDDI (Diffeomorphic Registration for Blip-Up blip-Down Diffusion Imaging) method for correcting echo planar imaging distortions. Neuroimage 2015; 106: 284-299. [PubMed: 25433212]

29. Optimization of non-linear image registration in AFNI. Proceedings of the XSEDE 2016; Miami, Florida. ACM.

30. Preacher KJ, Hayes AF. Asymptotic and resampling strategies for assessing and comparing indirect effects in multiple mediator models. Behavior Research Methods 2008; 40(3): 879-891. [PubMed: 18697684]

31. Efron B, Tibshirani RJ. An introduction to the bootstrap. Chapman \& Hall: Boca Raton, FL, 1993.

32. Cox RW, Chen G, Glen DR, Reynolds RC, Taylor PA. fMRI clustering and false-positive rates. Proceedings of the National Academy of Sciences of the United States of America 2017; 114(17): E3370-E3371. [PubMed: 28420798] 
33. Kraguljac NV, Anthony T, Skidmore FM, Marstrander JR, Morgan CJ, Reid M et al. Micro-and macrostructural white matter integrity in never-treated and currently unmedicated patients with schizophrenia and effects of short term antipsychotic treatment Biological Psychiatry: CNNI 2019; in press.

34. Reid MA, White DM, Kraguljac NV, Lahti AC. A combined diffusion tensor imaging and magnetic resonance spectroscopy study of patients with schizophrenia. Schizophr Res 2016; 170(2-3): 341-350. [PubMed: 26718333]

35. Baron RM, Kenny DA. The moderator-mediator variable distinction in social psychological research: conceptual, strategic, and statistical considerations. J Pers Soc Psychol 1986; 51(6): 1173-1182. [PubMed: 3806354]

36. Kelly S, Jahanshad N, Zalesky A, Kochunov P, Agartz I, Alloza C et al. Widespread white matter microstructural differences in schizophrenia across 4322 individuals: results from the ENIGMA Schizophrenia DTI Working Group. Mol Psychiatry 2018; 23(5): 1261-1269. [PubMed: 29038599]

37. Perkins DO, Gu H, Boteva K, Lieberman JA. Relationship between duration of untreated psychosis and outcome in first-episode schizophrenia: a critical review and meta-analysis. Am J Psychiatry 2005; 162(10): 1785-1804. [PubMed: 16199825]

38. Anderson KK, Rodrigues M, Mann K, Voineskos A, Mulsant BH, George TP et al. Minimal evidence that untreated psychosis damages brain structures: a systematic review. Schizophr Res 2015; 162(1-3): 222-233. [PubMed: 25649287]

39. Lee SW, Lee A, Choi TK, Kim B, Lee KS, Bang M et al. White matter abnormalities of the tapetum and their associations with duration of untreated psychosis and symptom severity in first-episode psychosis. Schizophr Res 2018; 201: 437-438. [PubMed: 29895415]

40. Filippi M, Canu E, Gasparotti R, Agosta F, Valsecchi P, Lodoli G et al. Patterns of brain structural changes in first-contact, antipsychotic drug-naive patients with schizophrenia. AJNR Am J Neuroradiol 2014; 35(1): 30-37. [PubMed: 23744689]

41. Cropley VL, Klauser P, Lenroot RK, Bruggemann J, Sundram S, Bousman C et al. Accelerated Gray and White Matter Deterioration With Age in Schizophrenia. Am J Psychiatry 2017; 174(3): 286-295. [PubMed: 27919183]

42. Lee S-W, Kim S-W, Lee S-H. White matter alterations related to duration of untreated periods in patients with recent-onset psychosis. Int J Neuropsychopharmacol 2016; 19: 70.

43. Squarcina L, Bellani M, Rossetti MG, Perlini C, Delvecchio G, Dusi N et al. Similar white matter changes in schizophrenia and bipolar disorder: A tract-based spatial statistics study. PLoS One 2017; 12(6): e0178089. [PubMed: 28658249]

44. Reis Marques T, Taylor H, Chaddock C, Dell'acqua F, Handley R, Reinders AA et al. White matter integrity as a predictor of response to treatment in first episode psychosis. Brain 2014; 137(Pt 1): 172-182. [PubMed: 24253201]

45. Crespo-Facorro B, Roiz-Santianez R, Pelayo-Teran JM, Gonzalez-Blanch C, Perez-Iglesias R, Gutierrez A et al. Caudate nucleus volume and its clinical and cognitive correlations in first episode schizophrenia. Schizophr Res 2007; 91(1-3): 87-96. [PubMed: 17306506]

46. Keshavan MS, Haas GL, Kahn CE, Aguilar E, Dick EL, Schooler NR et al. Superior temporal gyrus and the course of early schizophrenia: progressive, static, or reversible? J Psychiatr Res 1998; 32(3-4): 161-167. [PubMed: 9793869]

47. Choi MH, Na JE, Yoon YR, Lee HJ, Yoon S, Rhyu IJ et al. Role of Dopamine D2 Receptor in Stress-Induced Myelin Loss. Sci Rep 2017; 7(1): 11654. [PubMed: 28912499]

48. Roy K, Murtie JC, El-Khodor BF, Edgar N, Sardi SP, Hooks BM et al. Loss of erbB signaling in oligodendrocytes alters myelin and dopaminergic function, a potential mechanism for neuropsychiatric disorders. Proc Natl Acad Sci U S A 2007; 104(19): 8131-8136. [PubMed: 17483467]

49. Kraguljac NV, Morgan CJ, Reid MA, White DM, Jindal RD, Sivaraman S et al. A longitudinal magnetic resonance spectroscopy study investigating effects of risperidone in the anterior cingulate cortex and hippocampus in schizophrenia. Schizophr Res 2019.

50. de la Fuente-Sandoval C, Reyes-Madrigal F, Mao X, Leon-Ortiz P, Rodriguez-Mayoral O, Jung-Cook $\mathrm{H}$ et al. Prefrontal and Striatal Gamma-Aminobutyric Acid Levels and the Effect 
of Antipsychotic Treatment in First-Episode Psychosis Patients. Biol Psychiatry 2018; 83(6): 475483. [PubMed: 29132653]

51. Egerton A, Bhachu A, Merritt K, McQueen G, Szulc A, McGuire P. Effects of Antipsychotic Administration on Brain Glutamate in Schizophrenia: A Systematic Review of Longitudinal (1)HMRS Studies. Front Psychiatry 2017; 8: 66. [PubMed: 28503156]

52. Egerton A, Broberg BV, Van Haren N, Merritt K, Barker GJ, Lythgoe DJ et al. Response to initial antipsychotic treatment in first episode psychosis is related to anterior cingulate glutamate levels: a multicentre (1)H-MRS study (OPTiMiSE). Mol Psychiatry 2018.

53. de la Fuente-Sandoval C, Leon-Ortiz P, Azcarraga M, Stephano S, Favila R, Diaz-Galvis L et al. Glutamate levels in the associative striatum before and after 4 weeks of antipsychotic treatment in first-episode psychosis: a longitudinal proton magnetic resonance spectroscopy study. JAMA Psychiatry 2013; 70(10): 1057-1066. [PubMed: 23966023]

54. Matute C, Alberdi E, Domercq M, Sanchez-Gomez MV, Perez-Samartin A, Rodriguez-Antiguedad A et al. Excitotoxic damage to white matter. J Anat 2007; 210(6): 693-702. [PubMed: 17504270]

55. Hassel B, Boldingh KA, Narvesen C, Iversen EG, Skrede KK. Glutamate transport, glutamine synthetase and phosphate-activated glutaminase in rat CNS white matter. A quantitative study. $\mathrm{J}$ Neurochem 2003; 87(1): 230-237. [PubMed: 12969269]

56. Rabinowitz J, Levine SZ, Barkai O, Davidov O. Dropout rates in randomized clinical trials of antipsychotics: a meta-analysis comparing first- and second-generation drugs and an examination of the role of trial design features. Schizophr Bull 2009; 35(4): 775-788. [PubMed: 18303093]

57. Register-Brown K, Hong LE. Reliability and validity of methods for measuring the duration of untreated psychosis: a quantitative review and meta-analysis. Schizophr Res 2014; 160(1-3): 20 26. [PubMed: 25464915] 


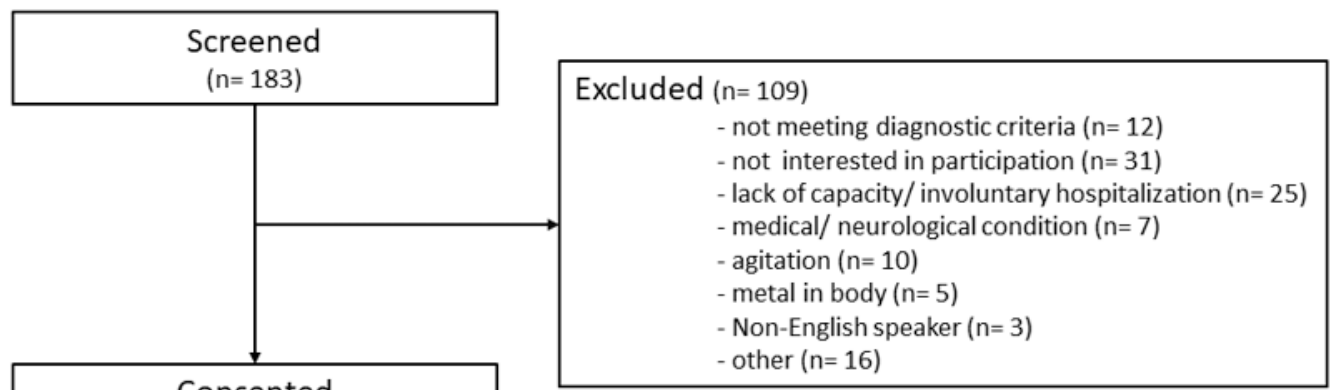

Consented $(n=74)$

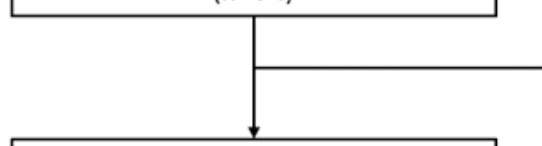

Entered into antipsychotic trial $(n=66)$

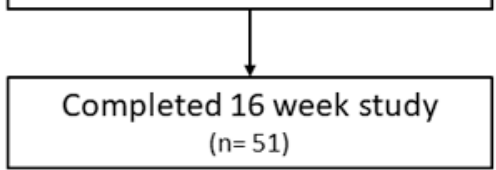

Figure 1:

CONSORT flow chart.
Dropped before start of antipsychotic trial $(n=8)$

- withdrew consent before scan $(n=2)$

- did not tolerate scan environment $(n=4)$

- other $(n=2)$ 

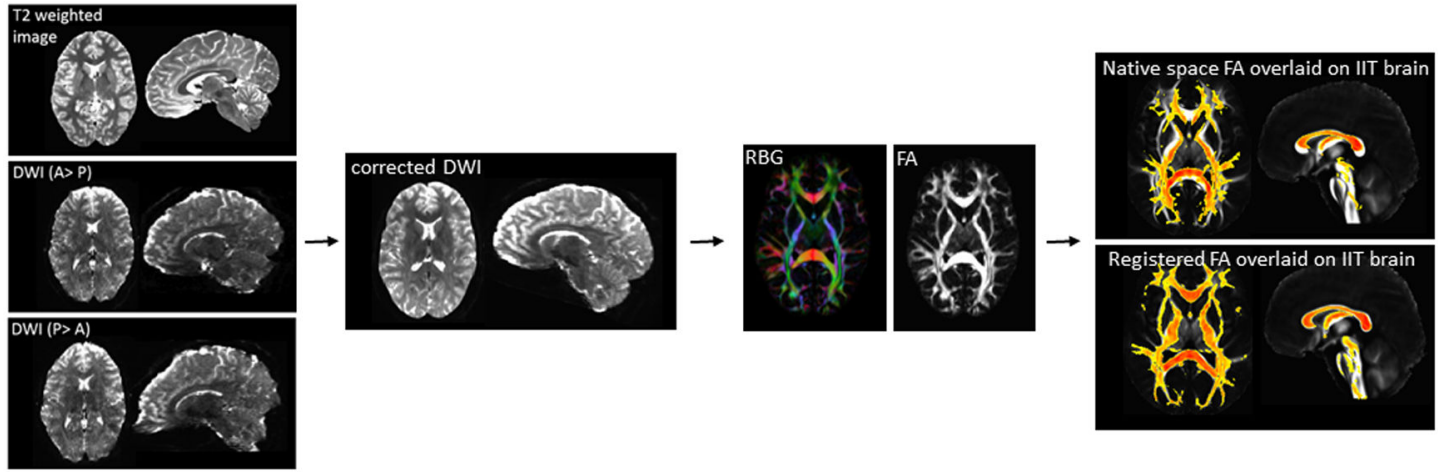

Figure 2:

Diffusion weighted imaging (DWI) data processing pipeline. A: anterior; p: posterior; RBG: red blue and green color map; FA fractional anisotropy; IIT: Illinois institute for technology. 
A

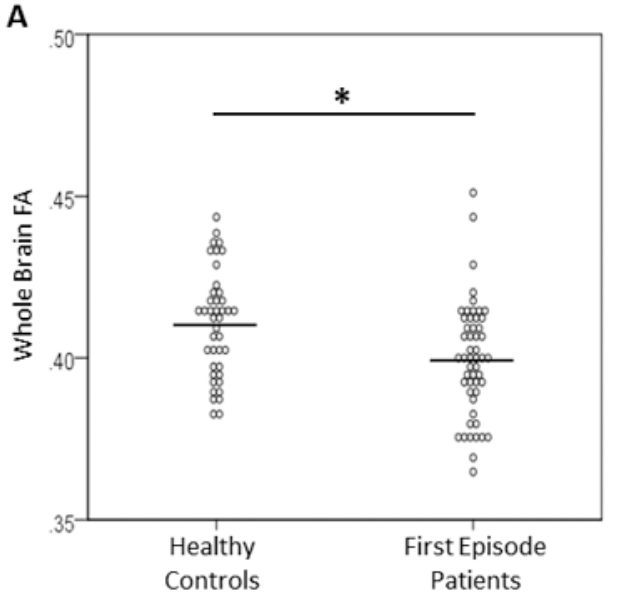

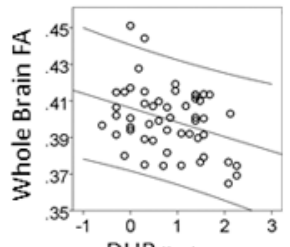

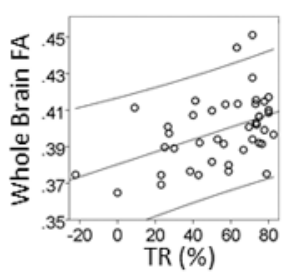

\section{항}

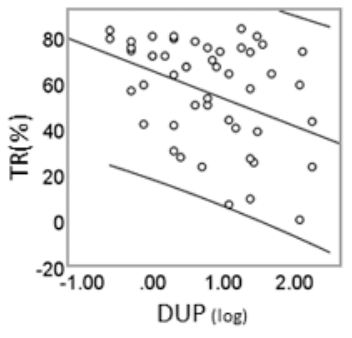

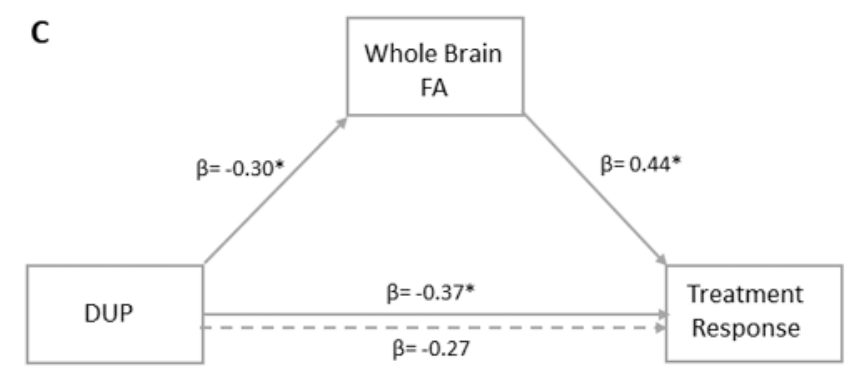

Figure 3:

White matter integrity, duration of untreated psychosis and response to antipsychotic treatment. A. At baseline, whole brain fractional anisotropy (FA) is lower in first episode psychosis patients compared to healthy controls. Dots represent individual measurements, the lines represent the group means. B. Top left panel. In first episode patients, whole brain FA is inversely related to duration of untreated psychosis (DUP; note that values are log transformed). Bottom left panel. In first episode patients, whole brain FA at baseline predicts subsequent response to antipsychotic treatment (TR). Right panel. Greater DUP is associated with poorer subsequent TR. C. Mediation model, where DUP is the independent variable, treatment response is the dependent variable and whole brain FA is the mediator. Numbers indicate standardized beta coefficient $(\beta)$ for regression analyses. 

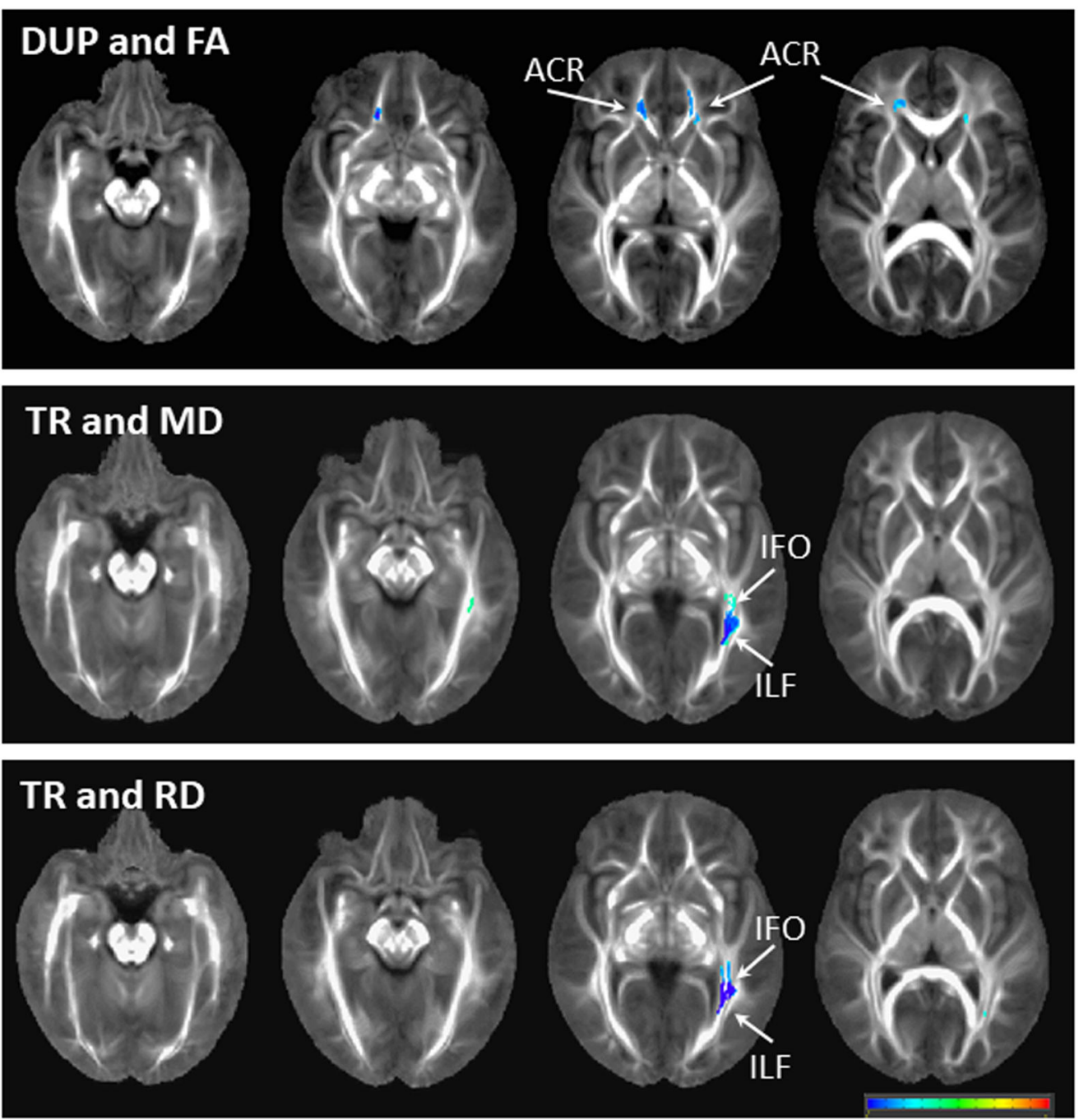

Figure 4:

Regional patterns of associations between white matter integrity and duration of untreated psychosis (DUP) as well as to response to antipsychotic treatment (TR). Images are overlaid on the IIT4 atlas. Blue colors reflect a negative association between white matter values and clinical values. Abbreviations: FA fractional anisotropy, MD mean diffusivity, RD radial diffusivity, ACR anterior corona radiata, IFO inferior fronto-occipital fasciculus, ILF inferior longitudinal fasciculus. 
Table:

Demographics, clinical measures, and motion parameters ${ }^{a}$

\begin{tabular}{|c|c|c|c|c|}
\hline & $\operatorname{FEP}(n=66)$ & HC $(n=45)$ & $t / X^{2} / F$ & p value \\
\hline \multicolumn{5}{|l|}{ Demographic variables } \\
\hline Gender ( $\%$ male $)$ & $65.2 \%$ & $64.4 \%$ & & \\
\hline Age & $23.83(6.21)$ & $24.78(6.24)$ & 0.785 & 0.43 \\
\hline Parental Occupation $^{b}$ & $5.95(4.83)$ & $4.22(4.06)$ & 11.68 & 0.63 \\
\hline \multicolumn{5}{|l|}{ Clinical variables } \\
\hline \multicolumn{5}{|l|}{ Diagnosis } \\
\hline Schizophrenia & 32 & & & \\
\hline Schizoaffective Disorder & 12 & & & \\
\hline Schizophreniform Disorder & 3 & & & \\
\hline Brief Psychotic Disorder & 2 & & & \\
\hline Bipolar Disorder with psychosis & 3 & & & \\
\hline Major Depression with psychosis & 2 & & & \\
\hline Unspecified Psychosis & 12 & & & \\
\hline $\begin{array}{l}\text { Duration of untreated psychosis } \\
\text { [in months; range, median, mean, } \\
\text { (SD)] }\end{array}$ & $0.3-180,6.00,20.57,(38.87)$ & & & \\
\hline UDS +cannabis (\%) & 31.8 & & & \\
\hline Risperidone dose at week $16^{c}$ & $4.11(2.66)$ & & & \\
\hline \multicolumn{5}{|l|}{$\mathrm{BPRS}^{d}$} \\
\hline \multicolumn{5}{|l|}{ Baseline } \\
\hline Total & $50.85(11.62)$ & & & \\
\hline Positive & $15.88(4.18)$ & & & \\
\hline Negative & $6.00(3.23)$ & & & \\
\hline \multicolumn{5}{|l|}{ BPRS } \\
\hline \multicolumn{5}{|l|}{ Week 16} \\
\hline Total & $29.94(5.88)$ & & & \\
\hline Positive & $6.38(2.68)$ & & & \\
\hline Negative & $5.69(2.83)$ & & & \\
\hline \multicolumn{5}{|l|}{ RBANS $^{e}$} \\
\hline Total index & $76.16(16.05)$ & $94.41(10.94)$ & 6.66 & $<.01$ \\
\hline Immediate memory & $81.79(18.55)$ & $99.64(17.05)$ & 4.80 & $<.01$ \\
\hline Visuospatial & $77.12(17.73)$ & $99.28(14.53)$ & 2.76 & $<.01$ \\
\hline Language & $83.98(15.49)$ & $99.28(14.53)$ & 4.89 & $<.01$ \\
\hline Attention span & $80.19(17.18)$ & $100.95(17.30)$ & 5.81 & $<.01$ \\
\hline Delayed memory & $78.59(14.89)$ & $92.36(9.10)$ & 5.16 & $<.01$ \\
\hline \multicolumn{5}{|l|}{ Scan quality data } \\
\hline \multicolumn{5}{|l|}{ DWI } \\
\hline RMS absolute motion (mm) & $0.40(0.24)$ & $0.34(0.22)$ & -1.16 & 0.25 \\
\hline
\end{tabular}




\begin{tabular}{lllll}
\hline & FEP $(\mathbf{n}=\mathbf{6 6})$ & HC $(\mathbf{n}=\mathbf{4 5})$ & $\mathbf{t} / \mathbf{X}^{\mathbf{2}} / \mathbf{F}$ & $\mathbf{p}$ value \\
\hline RMS relative motion $(\mathrm{mm})$ & $0.007(0.007)$ & $0.006(0.005)$ & -1.05 & 0.30 \\
\hline
\end{tabular}

FEP, first episode psychosis patient; HC, healthy control; UDS, urine drug screen; DWI, diffusion weighted image; DWI, diffusion weighted imaging; RMS, root mean square of the six motion parameters (translations and rotations)

${ }^{a}$ Mean (SD) unless indicated otherwise

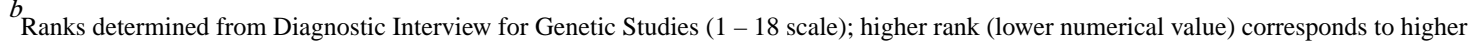
socioeconomic status

${ }^{c}$ Two subjects were switched to aripiprazole prior to week 16 (10mg daily and $20 \mathrm{mg}$ daily respectively), and are not included in the average dose

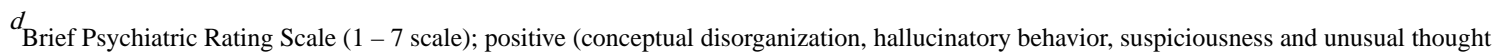
content); negative (emotional withdrawal, motor retardation, and blunted affect)

${ }^{e}$ Repeatable Battery for the Assessment of Neuropsychological Status 